\title{
A Randomized Trial of Ligustrazini Hydrochlorioi in the Treatment of Viral Encephalitis in Children
}

\author{
Jiao $\mathrm{F}^{1}$, Guo $\mathrm{G}^{2}$, Lin $\mathrm{J}^{3}$, Cui $\mathbf{W}^{4}$, Li H \\ ${ }^{1}$ Prof. Fuyong Jiao, MD, ${ }^{2} \mathrm{Dr}$. Xiangyang Guo, MD, ${ }^{3} \mathrm{Dr}$. Jin Lin, MD from the Department of Paediatrics, Shaanxi \\ Provincial People's Hospital, Xi'an-710068, China, ${ }^{4}$ Dr. Wei Cui, ${ }^{5}$ Dr. Huirong Li from the Department of Paediatrics, The \\ Second Teaching Hospital, Yan'an Medical College, Suide, Shaanxi, China.
}

Address for correspondence: Dr. Fuyong Jiao, E-mail: jiaofy@yeah.net

\begin{abstract}
Introduction: Chuanxiong is a herb used in traditional Chinese medicine for the Treatment of viral encephalitis. In animal studies it has shown to inhibit the synthesis and activity of Thromboxane (TXA2) and abate the imbalance between Thromboxane (TXA2) and Prostacyclin (PGI2). As a result, cerebral edema, ischemia and hypoxia could be improved. The aim of this study is to evaluate its effect in the treatment of viral encephalitis in children. Methodology: Ninety-nine patients with viral encephalitis were randomly divided into two groups. Ligustrazini Hydrochlorioi (LH) consisting of 51 cases (males 30 , females 21; age 5 years and nine month \pm 8 years and 2 month) was given LH $4 \mathrm{mg} / \mathrm{kg}$ per day in 100 $300 \mathrm{mls}$ of $10 \%$ glucose and infused intravenously over a three to four hour period, for 7 days as a course of treatment. A control groups of 48 cases(males 31, females 17; age 5 years and three month \pm 4 years and three month) received the conventional treatment of Vitamin $C(2.0-3.0 \mathrm{~g})$, Coenzyme $A(100 \mathrm{u})$ and Adenosine Triphosphate(ATP)(40mg) in $100-300 \mathrm{mls}$ of $10 \%$ glucose infused intravenously daily for 7 days. Results: The total response rate in the LH group and the control group were $94.12 \%$ and $68.75 \%$ respectively $(u=3.271 \mathrm{p}<0.05)$. The average time to improvement was $4.29 \pm 1.41$ days and $7.31 \pm 2.66$ days respectively. No adverse effect was observed in both groups. Conclusion: We conclude that LH is an effective, safe and well tolerated treatment for children encephalitis.
\end{abstract}

Key words: Cerebral Oedema, Encephalitis, Ligustrazini Hydrochlorioi

\section{Introduction}

$\mathrm{V}$ al encephalitis is a life-threatening condition with high mortality as well as significant sequelae. Although acyclovir and vidarabine have proven efficacy in the treatment of herpes simplex encephalitis, viral encephalitis is a potentially fatal viral infectious disease and has the different prognosis of the disease, mortality and sequelae still remain problematic, including the neurological sequelae, lesions in the limb and even death ${ }^{1,2,3}$. Moreover conventional treatment is available only for herpes simplex encephalitis, which is very expensive. Therefore research of an effective inexpensive and well tolerated medicine is very important for the treatment of viral encephalitis. Chuanxiong (an extract of the plant Ligusticum Wallichii Franch) has been reported in Chinese literature to be effective in the treatment of acute cerebral infarction and viral encephalitis. Investigations in China have shown that Chuanxiong can inhibit the synthesis and activity of Thromboxane A2 (TXA2) and abate the imbalance between Thromboxane A2 (TXA2) and Prostacyclin (PGI2), relieve cerebral edema, improve cerebral microcirculatory disturbance, enlarge volume of blood flow, inhibit accumulation of erythrocyte and platelet, increase speed of blood flow, expand contracted artery. It can also promote recovery of degenerated nerve dendritic cell and relieve lesion of ultrastructure of cell through electric microscopy; as a result, cerebral ischemia, edema and hypoxia are improved. The imbalance between TXA2 and PGI2 and demyelination or vasculitis are believed to an important factor in the development of primary or secondary brain 
injury and inflammation ${ }^{4,5,6,7}$. Ligustrazini hydrochlorioi (LH) could be especially valuable for viral encephalitis in which the aim of treatment is to reduce cerebral ischemia, edema, oxygen radical injury and hypoxia. Previous studies that were done in adults with dose regime of $40-100 \mathrm{mg} / \mathrm{per}$ day, but there has been no detailed prospective evaluation of $\mathrm{LH}$ in children. We have therefore compared the responses of intravenous $\mathrm{LH}$ therapy with conventional treatment in childhood with viral encephalitis.

\section{Materials and Methods}

This study was carried out in the Second Teaching Hospital of Yan'an Medical College and the study was approved by the Ethics Committee of the hospital. Ninety-nine infants and young children (61 males and 38 females) were included in this study of the treatment of viral encephalitis. Their ages varied from 8 months to 14 yeas. The age range in the LH group was $5.98 \pm 8.21$ years and that of the control group was $5.38 \pm 7.33$ years. The two groups with viral encephalitis were comparable in terms of clinical features and laboratory indices on entry to the study (Table I).

Patients had to satisfy the following criteria: They had to have clinical manifestations of viral encephalitis (Namely: fever, headache, dizziness, vomiting, seizures, convulsions, coma, stiff back, stiff neck, Brudzinski's and Kernig's signs positive). The diagnosis was established by a combination of the clinical features, laboratory and EEG examinations. The patients were randomly assigned into two groups. Fifty-one cases were given $\mathrm{LH}(40 \mathrm{mg} / 2 \mathrm{mls}$ ampoule. Manufactured by the pharmaceutical factory; Zhangjiang, Guongdong, People's Republic of China) at a dosage of $4 \mathrm{mg} / \mathrm{kg}$ per day. The LH preparation was diluted in 100-300 mls of $10 \%$ glucose solution and infused intravenously over a three to five hours period, once a day for seven days as a course of treatment. The other 48 cases which made up the control group, received the convention treatment of Vitamin C 2.0-3.0g per day, Coenzyme A and Adenosine Triphosphate(ATP) in $100-300 \mathrm{mls}$ of $10 \%$ glucose solution and infused intravenously once a day for seven days. All the cases simultaneously received the standard protocol permitted for the treatment of patients with acute viral encephalitis, for example, intravenous fluids antipyretics, osmotic agents and anticonvulsions were given as indicated clinical. Vital signs, neurological and clinical observations were recorded twice a day for seven days. The side effects were noted by the doctors and nurses during the treatment.

The response of the patients was based on the following criteria:

a. Very effective: the patients did not have any more convulsions and coma after 3-7 days, EEG and /or CSF was completely normal on discharge.

b. Slightly effective: the patients did not have any more convulsions and coma after 3-7 days, EEG and /or CSF was not completely normal on discharge.

c. Not effective: the patients did not have shown any improvement in the convulsions and coma after 3-7 days, EEG and /or CSF remained abnormal.

Statistical Analysis: Means of clinical features where compared by percent, student test and differences between groups where compared by Ridit (relative to an identified distribution) analysis.

\section{Results}

There were no significant differences between the two groups with respect to sex distribution, age and evaluation criteria. The two groups were similar and all important baseline variables (Table 1). The outcome variables of the $\mathrm{LH}$ group were compared to those of control group (Table 2). In comparison with the control group, the patients treated with $\mathrm{LH}$ had significantly less convulsions and comas. There were statistically significant differences between the two groups including the very effective and slightly effective responses $(U=3.154, p<0.05)$. The total effective rate of the $\mathrm{LH}$ group and control group were $94.12 \%$ and $68 \%, 75 \%$ respectively $(U=3.271, p<0.05)$.

Side-effects: Nine patients had transient flushing in the LH group, but no patients were withdrawn from the study. No other adverse effects were noted. 
Table 1: Showing Characteristica of Randomised Patients.

\begin{tabular}{|l|c|c|}
\hline Characteristics & LH Group (N=51) & Control Group (N=48) \\
\hline Male & 30 & 31 \\
\hline Female & 21 & 17 \\
\hline M:F Ratio & 1.43 & $5.38 \pm 7.33$ \\
\hline Mean Age & $5.98 \pm 8.21$ & $8(16.67 \%)$ \\
\hline Lethargy & $5(9.80 \%)$ & $11(22.92 \%)$ \\
\hline Delirium & $7(13.72 \%)$ & $6(12.50 \%)$ \\
\hline Coma & $10(19.60 \%)$ & $15(31.28 \%)$ \\
\hline Convulsion & $36(70.58 \%)$ & $32(62.50 \%)$ \\
\hline Duration of seizures & & $15(31.25 \%)$ \\
\hline$<10$ minutes & $32(62.75 \%)$ & $3(6.25 \%)$ \\
\hline $10-30$ mintues & $13(25.49 \%)$ & $39(81.25 \%)$ \\
\hline$>30$ mintues & $6(11.76 \%)$ & $22(45.83 \%)$ \\
\hline EEG abnormalities & $41(80.39 \%)$ & $25(49.01 \%)$ \\
\hline CSF abnormalities & & \\
\hline
\end{tabular}

LH: Ligustrazini Hydrochlorioi

Table 2: Showing Therapeutic Response of Two Groups.

\begin{tabular}{|l|c|c|}
\hline Response & LH Group (N=51) & Control Group (N=48) \\
\hline Very Effective & $39(76.47 \%)$ & $21(43.75 \%)$ \\
\hline Slightly Effective & $9(17.64 \%)$ & $12(25.00 \%)$ \\
\hline Not Effective & $3(5.88 \%)$ & $3(13.25 \%)$ \\
\hline Effective(Total) & $48(94.12 \%)$ & $9(68.75 \%)$ \\
\hline Neurological Sequelae & & $21(43.75 \%)$ \\
\hline Paralysis of limb & $4(7.84 \%)$ & $17(35.41 \%)$ \\
\hline Memory impairment & $13(25.49 \%)$ & $22(45.83 \%)$ \\
\hline Anosmia & $19(37.25 \%)$ & $26(54.16 \%)$ \\
\hline Dysphasia & $12(23.53 \%)$ & $8(16.67 \%)$ \\
\hline $\begin{array}{l}\text { Personality and Behavioural } \\
\text { abnormalities }\end{array}$ & $18(35.29 \%)$ & $15.78 \pm 8.17$ days \\
\hline Mortality & $3(5.88 \%)$ & $7.31 \pm 2.66$ days \\
\hline Average time of Hospitalization & $13.29 \pm 5.44$ days & \\
\hline $\begin{array}{l}\text { Average duration of } \\
\text { Rx before effect occurs }\end{array}$ & $4.29 \pm 1.41$ days & \\
\hline & & \\
\hline
\end{tabular}

\section{LH: Ligustrazini Hydrochlorioi}

\section{Discussion}

Encephalitis is uncommon but can be serious and potentially fatal. It is usually caused by viruses such as arboviruese, herpesviruses, and enteroviruses. Viral replication and/or oxygen radical injury in the central neuronal tissue causes cell damage and surrounding inflammation ensues. Although the disease carries an extremely grave prognosis, there is no specific treatment. Various treatments including convalescent plasma, antiviral agents and interferon have been tried but without demonstrable benefits. Some authors have claimed favorable results with corticosteroids.
Others, however, could not confirm the beneficial effects or even reported adverse effects ${ }^{6,7}$. Ligusticum Wallischii Franch is a traditional Chinese medicine. It is an effective treatment for acute cerebral infarction and viral encephalitis. According to clinical observations, theory, if traditional Chinese medicine, and previous pharmacological studies in animals; LH was shown to activate blood circulation and dispersing or stasis. It has been proved in experiments on animals that $\mathrm{LH}$ can inhibit the synthesis and activity of Thromboxane (TXA2) and abate the imbalance between TXA2 and PGI2. 
Thromboxane (TXA2) and PGI2 are important factors in the development of primary or secondary brain injury, edema, oxygen radical injury and inflammation. The present studies in China confirm the beneficial effect. LH could be especially valuable for viral encephalitis in which the aim of treatment is to reduce cerebral edema, oxygen radical injury, ischemia and hypoxia. It has been shown in patients with pregnancy induced hypertension that $\mathrm{LH}$ can decrease the TXB2/6-kto-PGF1 alpha and release vasoconstriction ${ }^{8}$. Animal studies have shown that $\mathrm{LH}$ has protective effects against ischemia and reperfusion injury of rat liver by scanvenging free radicals and improve hepatic microcirculation ${ }^{9}$. This radomized and controlled trial demonstrated that treatment with $\mathrm{LH}$ leads to significant improvement of the occurrence of convulsion and comas. The three aspects; duration of hospitalization, duration of treatment before effects, and overall effective rate, in the LH is a safe, well tolerated, is relatively inexpensive, and widely applicable treatment for childhood encephalitis.

\section{Acknowledgement}

We would like to thank Dr.Rita Sung, Department of Paediatrics, Prince of Wales Hospital, Chinese University of Hong Kong for her advice in the preparation of the manuscript.

Funding: Nil.

Conflict of Interest: None.

\section{References}

1. B. Skoldenberg. Herpes simplex encephalitis, Scand J Infect. Dis Suppl 1996;100:8-13.

2. Andersson T., Olsson J., Forkman A. et al: Herpes simplex encephalitis-a therapeutic failure with acyclovir, Lakartidniingen 1987;84:3390-3393. [Swidish].
3. McGrath N, Anderson NE, Croxson MC, et al: Herpes simplex encephalitis treated with acylovir: diagnosis and long term outcome, J Neurol Neurosurg Psychiatr 1997;63:321-326.

4. Tong Y.Z, Zhu S.X, Chen H.L, et al: Comparison of efficacy between Mailuoning and Ligustrazini Hydrochlorioi in treating acute cerebral infarction in 40 patients each. New Drugs and Clinical Remedies 1992;4:207-208.

5. Fei Z, Bia A N, Liu N S, et al: The alteration of Thromboxane A2 and Prostacyxlin contents and effects of Ligustrazini Hydrochlorioi after rat head injury. Chinese Joural of Traumatol 1992;2:85-87.

6. Chen D.R, don W.Y, Xiu C.H, et al: Comparative study of Ligustrazini Hydrichlorioi and Dextran 40 in treatment of acute cerebral infarction. Chin Med Sci J 1993;2:122-126.

7. Jiang G.H, Li Z.J, Y M Cao, et al: The effects of Ligustrazini Hydrochloroi on cerebral edema in rabbit. Chinese Journal of Pediatr 1989;5:300.

8. Liu S.Y, Xu Y.Y, Zhu J.Y. The effects of Salvia Miltirrhizae Bge and Liguatriazini Hydrochloioi on Thromboxane A2 and Prostacyclin in pregnancy induced hypertension. Chung Hua Fu Chan Ko Tsa Chin.1994; 11:648-650.

9. Wu W, Qiu F. Experimental study on ischemia and reperfusion injury of rat liver and effects of Ligustrazini Hydrochlorioi and Salvia compound. Chin Med Sci J.1994; 3:162-166. 